\title{
A Narrowing of the Phenotypic Diversity Range after Large Rearrangements of the Karyotype in Salmonidae: The Relationship between Saltational Genome Rearrangements and Gradual Adaptive Evolution
}

\author{
Alexander A. Makhrov ${ }^{1,2}$ (ii \\ 1 A.N. Severtsov Institute of Ecology and Evolution of Russian Academy of Sciences, Moscow 119071, Russia; \\ makhrov12@mail.ru \\ 2 Institute of Biophysics of Siberian Branch of Federal Research Center "Krasnoyarsk Science Center" \\ of Russian Academy of Sciences, Akademgorodok, 50/50, Krasnoyarsk 660036, Russia
}

Received: 24 August 2017; Accepted: 24 October 2017; Published: 27 October 2017

\begin{abstract}
The problem of how a gradual development of ecological and morphological adaptations combines with large genome rearrangements, which have been found to occur in the phylogeny of many groups of organisms, is a matter of discussion in the literature. The objective of this work was to study the problem with the example of salmonids, whose evolution included at least six events of multiple chromosome fusions. Large karyotype rearrangements are associated with a decrease in ecological and morphological diversity in salmonids. In the above example, genome rearrangements seem to distort the function of the genetic systems that are responsible for the occurrence of certain ecological forms in salmonids.
\end{abstract}

Keywords: evolution; karyotype; genome; morphology; ecology

\section{Introduction}

During recent years, studies of evolutionary developmental morphology (MorphoEvoDevo), genetics and ecology have converged in the holistic approach [1-3]. However, there are still some unresolved problems in the field of research.

Particularly, large genome rearrangements unrelated to distant hybridization have been found to occur in many groups of organisms during their phylogeny. The set includes autopolyploidy [4], simultaneous multiple chromosome fusions [5], and mass transpositions of mobile genetic elements [6]. The findings are in conflict with the conventional Darwinian views of gradual adaptive evolution, as has been noted in several monographs [7-10]. It is clear that large genome rearrangements would dramatically change the genetic systems formed in earlier evolution, and thus distort respective adaptations in many cases.

How are large genome rearrangements compatible with a gradual development of adaptations? The problem is extremely difficult to solve, because morphological or ecological features of a group originating via a genome rearrangement are not necessarily a consequence of the rearrangement. To understand how morphological and ecological traits change as a result of large genome rearrangements, it is necessary to study several cases where rearrangements of the same type occur in related organisms.

Such studies are possible, in particular, for autopolyploidy, which has occurred many times in evolution. An analysis of its evolutionary consequences in plants [11] and teleosts [12] has led to the idea of autopolyploidy as a nearly neutral process. Experimental data available for salmonids 
agree with the idea, apart from sterility in triploids $[13,14]$. However, functional divergence of many duplicated genes as a result of long-term evolution has been observed in polyploid groups, including those of salmonids [15-17].

Other chromosome rearrangements are usually deleterious for their carriers (for a review, see [18]). It is therefore difficult to search for neutral or adaptively advantageous chromosome rearrangements in an experimental setting. Yet such rearrangements, including large ones, have certainly occurred in evolution.

For instance, simultaneous multiple chromosome fusions have repeatedly taken place in several groups of organisms [5]. Several such events have occurred in the evolution of salmonids. The group has two main karyotype categories, type A of karyotypes with approximately 80 chromosomes and type B of karyotypes with approximately 60 chromosomes [19]. There have been at least six events where a type B karyotype originated from a type A karyotype in the evolution of salmonids [20,21].

Balon [22] has associated a decrease in chromosome number with specialization in salmonids. A similar assessment was proposed by Qumsiyeh [23] with mammals. However, Pavlov and Osinov [24] have criticized the assumptions of Balon [22] in their comprehensive study.

Based on published data, it is discussed belowwhether large karyotype rearrangements occurring independently in different phylogenetic lineages of salmonids have similarly affected their morphological and ecological diversity in a regular manner. Salmonids live in a variety of habitats, from small creeks to oceans, and are known to include a variety of ecological forms. However, the number of ecological forms differs among different salmonid species (Table 1). The species additionally vary in their ability to survive after spawning. The ability to colonize and to adapt to new habitats is another important, though rarely evaluated, sign of their phenotypic plasticity.

Table 1. Types of anadromous and resident life strategy in Salmoninae ([25], with addition) and their chromosome number (2n) [19,26-28].

\begin{tabular}{|c|c|c|c|c|c|c|c|c|c|c|c|c|c|c|c|}
\hline Species & \multicolumn{14}{|c|}{ Types of Strategy * } & $2 n$ \\
\hline Salmo trutta & + & + & + & + & + & + & - & + & + & + & + & - & + & + & $76-84$ \\
\hline Oncorhynchus gorbusha & + & - & - & - & - & - & - & - & - & - & - & - & - & - & $52-54$ \\
\hline Oncorhynchus keta & + & - & - & - & - & - & - & - & - & - & - & - & - & - & 74 \\
\hline Oncorhynchus kisutch & + & - & + & - & + & - & - & - & - & - & - & - & + & - & 60 \\
\hline Oncorhynchus tschawytcha & + & - & + & - & + & - & - & - & - & - & - & - & - & - & 68 \\
\hline Oncorhynchus masou & + & - & + & - & + & + & - & - & - & - & - & - & + & - & 66 \\
\hline Parasalmo mykiss & + & + & + & + & + & - & - & + & + & + & + & - & + & + & $58-65$ \\
\hline Parasalmo clarki & $?$ & + & - & + & + & + & - & + & - & + & $?$ & - & + & + & $64-68$ \\
\hline Parahucho perryi & + & - & - & - & - & - & - & + & - & + & - & - & - & + & 62 \\
\hline
\end{tabular}

* (1) Typically migratory (2) smolts initially feed in seawater, return for wintering to rivers, and only then finally go for feeding to the sea until maturation (3) jacks, (4) half-pounders (thousanders, postsmolt, pregrilse), (5) dwarf riverine males, (6) dwarf riverine females, (7) facultatively anadromous, (8) estuarine, (9) riverine-estuarine, (10) riverine, (11) resident males and females which having spawned in fresh water may make downstream migration to the sea and return already as migratory fish, (12) resident dwarf lacustrine Oncorhynchus nerka (predominantly males as a part of the stock of migratory form), (13) lacustrine, (14) lacustrine-riverine, ? - no precise data.

\section{Atlantic Salmon Salmo salar L.}

Atlantic salmon spawns in rivers of northeastern regions of North America and western and northern regions of Europe [29]. The species has $2 n=54-60$, NF (chromosome arms) $=72-74$ (for a review, see [26]). Atlantic salmon originated from an ancestor similar to the modern brown trout Salmo trutta on evidence of integrated data (for a review, see [30]). Salmo trutta has $2 \mathrm{n}=76-84$, 
$\mathrm{NF}=92-104$ (for a review, see [27]). Thus, fusions of several chromosomes accompanied the origin of Atlantic salmon [21,31].

Interestingly, the species $S$. trutta includes forms that have larger spawners and are similar in many ecological and morphological traits to Atlantic salmon, differing from the relatively small typical brown trout [30]. The most brilliant example is provided by Black Sea and Caspian Sea populations. The forms have been assigned to Salmo salar in several studies. Yet a karyotype analysis has allowed their exact species identification [30].

While substantial ecological diversity is characteristic of S. trutta, the majority of $S$. salar populations include only large individuals, which belong to the anadromous form, and dwarf males. Only a few populations include resident females, which are usually small in size [32]. The low frequency of these populations has been explained by the experimental finding that the early death rate in progeny of small-sized females is far greater than that of large-sized ones in S. salar ([33], and references therein).

Lower ecological plasticity of $S$. salar is seen well in South America, where the species has been introduced together with $S$. trutta. The latter has spread widely in the region, while $S$. salar is restricted to few large water systems [34].

As in all salmonids, S. salar is highly diverse, morphologically [35]. However, morphological diversity in S. salar is substantially lower than in the ancestral species S. trutta [32].

Thus, both large reorganization of the karyotype and a dramatic decrease in ecological and morphological diversity has occurred in $S$. salar during speciation. The karyotype reorganization might have considerably distorted the ability of small-sized freshwater females to mature, which was high in the ancestral species $S$. trutta. As is shown below, the hypothesis is supported by the fact that large karyotype rearrangements are associated with a decrease in ecological and morphological diversity in other salmonids.

\section{Pacific Salmonids (Oncorhynchus)}

The number of species in the genus has been the matter of long-standing discussion. There are six species in the group (Table 2), in our opinion, which has been grounded previously [27]. All species of the genus Oncorhynchus spawn in rivers discharging into the Northern Pacific Ocean and the adjacent part of Arctic ocean [36].

Table 2. Characteristics of species of the genus Oncorhynchus.

\begin{tabular}{ccc}
\hline Species & $\begin{array}{c}\text { Dwarf River Females and Males } \\
\text { ([25], with Addition) }\end{array}$ & $\begin{array}{c}\text { Multiple Spawning } \\
\text { ([37], with Addition) }\end{array}$ \\
\hline Pink salmon O. gorbuscha & No & No \\
\hline Chum salmon O. keta & No & No \\
\hline Sockeye salmon O. nerka & No & $\begin{array}{c}\text { Possible only in resident males. However, } \\
\text { females that survive spawning are found in } \\
\text { some lakes where resident O. nerka has been } \\
\text { introduced [38] }\end{array}$ \\
\hline Coho salmon O. kisutch & Dwarf males present & Possible only in resident males \\
\hline Chinook salmon O. tshawytscha & Dwarf males present & Possible only in dwarf males \\
\hline Cherry salmon O. masou & $\begin{array}{c}\text { Dwarf males present; “female parr } \\
\text { maturation requires extremely favorable } \\
\text { growth conditions" ([39], p. 962) }\end{array}$ & Multiple spawning present \\
\hline
\end{tabular}

A low chromosome number is characteristic of the majority of Oncorhynchus species (Table 1), which thereby belong to type $\mathrm{B}$, according to the above classification [19]. The chum salmon O. keta is the only exception, occupying an intermediate place between types A and B.

Females of the genus Oncorhynchus (with the exception of the cherry salmon Oncorhynchus masou) are single-time spawners (Table 2), while other salmonids (Salmo, Parasalmo, Salvelinus, Parahucho, 
Hucho, Brachymystax) are able to spawn more than once [37]. Of the 14 life-history strategies known for salmonids, only five have been observed in Oncorhynchus [25]. The capability of acclimatization is poor in salmons of the genus Oncorhynchus as compared with other salmonids [36]. Subspecies have not been recognized in the majority of the Oncorhynchus species, O. masou being the only exception [40].

Thus, a combination of a relatively low chromosome number with a decrease in ecological and morphological intraspecific diversity is characteristic of Oncorhynchus species.

\section{Pacific Trouts (Parasalmo)}

Parasalmo found in tributaries of the North Pacific, and is commonly included in the genus Oncorhynchus. Parasalmo species have relatively low chromosome numbers, 56-68, with NF $=104-106$ (for a review, see $[19,27,28]$. However, there are no signs of a decrease in morphological and ecological diversity in the group. In particular, the rainbow trout Parasalmo mykiss is one of the most plastic salmonid species, has a broad natural geographic range in the North Pacific (monograph: [41]), and has successfully been introduced into many rivers and lakes in various regions of the globe [36].

According to Balon [22], pedomorphosis accompanied the origin of Parasalmo and led to despecialization in this evolutionary branch. Whichever the case, large chromosome rearrangements do not always restrict morphological and ecological diversity in salmonids.

\section{Long-Finned Charr Salvethymus svetovidovi}

This species is found only in the El'gygytgyn Lake of the Chukchi Peninsula (Russia), and is the only species of the genus Salvethymus [42], although the independent status of this genus is strongly doubted now $[43,44]$. The Salvethymus svetovidovi karyotype consists of 56 chromosomes with NF $=98$ [28]. Arctic charrs of the genus Salvelinus, which are the closest relatives to S. svetovidovi, have 76-86 chromosomes with NF $=98-100$ [28]. Thus, substantial karyotype changes in the form of multiple chromosome fusions accompanied the origin of $S$. svetovidovi.

It is important to note that some morphological features of $S$. svetovidovi (a great number of gill rakers and reduction of certain bones) are still expressed, but to a lesser extent in char forms from Trans-Baikal lakes [45]. Like in the case of $S$. salar, a large karyotype rearrangement occurring in the course of speciation might have allowed $S$. svetovidovi to preserve only part of the morphological diversity spectrum characteristic of its ancestor belonging to the genus Salvelinus. Morphological and ecological diversity is extremely high in Salvelinus (Table 1; for a review, see [46-49]).

The species S. svetovidovi includes only one population, and has never been the subject of experimental research [50]; the stability of its morphological and ecological traits is therefore difficult to estimate. Yet the form seems to be conserved. The form diverged to a great extent from two sympatric forms of the genus Salvelinus, and its divergence was allopatric, as molecular genetic studies have shown [44]. It is noteworthy that the two forms have somehow found their way into the El'gygytgyn Lake, while S. svetovidovi has not spread to other lakes.

\section{Sakhalin Taimen Parahucho perryi}

The Sakhalin taimen karyotype includes 62 chromosomes with NF $=100$ [19,28]. Different genera have been identified as sister groups of Parahucho by different molecular genetic methods, the set including Hucho [51,52], Salmo [53,54], and Salvelinus [52,53,55]. The chromosome number is far greater, approximately 80 , in all of these groups (for a review, see $[19,28]$. Thus, multiple chromosome fusions occurred during the formation of Parahucho perryi, which is the only species in the genus Parahucho.

The P. perryi species range is relatively small; spawning occurs in rivers of Sakhalin, the Kuril Islands, Hokkaido, and the Russian coast of the Sea of Japan [56]. Gritsenko [56] have summarized the data available for P. perryi and characterized the species as a phenotypically homogeneous, monotypic species. 
Ecological plasticity is also limited in P. perryi [56]. Distinct ecological forms are apparently lacking. The species uses river, lake, and estuarine waters for feeding [57-59], although capable of living in marine waters [60]. Dwarf spawners have not been mentioned for the species in the available literature.

Thus, a combination of a dramatic decrease in chromosome number with a relatively low morphological and ecological diversity is observed again in P. perryi.

\section{Broad Whitefish Coregonus nasus}

The diploid chromosome set includes approximately 80 chromosomes in the majority of coregonids, while the broad whitefish Coregonus nasus has $2 \mathrm{n}=58-60, \mathrm{NF}=92-98$ (for a review, see $[19,28])$.

In a monograph focusing on coregonid fishes, Reshetnikov ([61], p. 170) has characterized C. nasus as a relatively stable species wherein subspecies or geographical forms can hardly be isolated objectively. Broad whitefish usually lives in rivers and is not found in waters with a salinity higher than 9-15\%0 [61].

Coregonus lavaretus and C. clupeaformis, which are the closest relatives to C. nasus [62], have very high morphological and ecological diversity ([61,63-65]. In particular, either species forms morphologically different populations in different geographic regions, has anadromous forms, and quickly adapt to new habitats (Volga tributaries, the Lake Sevan in the Caucasus, etc.).

Thus, a relatively low chromosome number and relatively low phenotypic diversity are characteristic of $C$. nasus.

\section{Immobilization: An Association of Gradual and Saltational Evolution}

It is important to note that a decrease in ecological and phenotypic diversity is restricted to the widespread salmonid species and genera that have a lower chromosome number (Table 1). The fact strongly suggests that chromosome fusions can explain why phenotypic diversity declines over time in some species.

Species with a low morphological and ecological plasticity thus originated from species that included many ecological forms, with each form having its morphological specifics. Note that the phenomenon differs from specialization, which is adaptation to particular way of life. The phenomenon is not adaptive; it is known as immobilization, that is, a reduction of evolutionary plasticity [66]. Unfortunately, this interesting phenomenon has only recently come to be studied in detail [67].

It is important to understand that immobilization does not mean that the species becomes totally incapable of adaptation as a result. For example, pink salmon has almost lost the capability to form resident populations, but is still highly competitive in a marine environment.

Gradual evolution was apparently combined with saltational evolution in salmonids. First, numerous forms that differ in ecology and morphology arise via gradual adaptive evolution within an ancestral taxon. Then, the capability of producing such forms is lost, potentially as a result of chromosome fusions in some species.

Thus, gradual evolution is not necessarily an alternative to saltational evolution, but the two respective scenarios may complement each other. A new species undergoing a large genome rearrangement will, in fact, hardly survive if expressing absolutely new phenotypic characters, which are extremely unlikely to be adaptive. Yet the species may survive if expressing the ecological and morphological characters that arose in the ancestral lineage via gradual adaptive evolution; these characteristics may currently be seen in particular groups within the related species.

Cases of rather large chromosome rearrangements are considered here, but other changes of the genome are probably capable of immobilizing ecological and morphological characters as well. For instance, a deletion or loss of expression of a gene can eliminate the pathways that lead to alternative phenotypes $[68,69]$.

The genome is a complex system that includes many regulatory elements [70]. A large genome rearrangement is therefore unlikely to immediately result in a new adaptation (although they open 
new opportunities for adaptations to arise). Yet such a rearrangement can easily and immediately eliminate one of several discrete adaptive norms (alternative adaptive phenotypes).

In the above example, genome rearrangements seem to distort the function of the genetic systems that are responsible for the occurrence of certain ecological forms in salmonids. For instance, resident forms can hardly develop in Atlantic salmon. Dysfunction of genetic systems is not an obligatory consequence of genome rearrangements; e.g., no sign of a decrease in morphological and ecological plasticity is seen in Parasalmo.

Immobilization, described above, yields organisms that display robustness; that is, lack of phenotypic variation in several characters. This robustness may favor adaptation in certain cases [71].

\section{Conclusions}

The examples known in salmonids demonstrate that large karyotype rearrangements are may be capable of reducing ecological and morphological diversity. It is clear from the above that gradual (adaptive) and saltational (nonadaptive) modes of evolution are not necessarily alternative. A plausible evolutionary scenario is that adaptations arise in the course of gradual evolution and are then fixed as a result of a genome rearrangement. The exact molecular mechanisms underlying the saltational evolution described here related to chromosome fusions is yet to be determined, and will be an important avenue of investigation for future studies.

Acknowledgments: I am grateful to Yu. P. Altukhov, V. S. Artamonova, E. A. Dorofeeva and Yu. P. Zelinsky for fruitful discussion of the problems considered in this work and T. Tkacheva for translation. The work was supported by a grant from the Russian Science Foundation No. 16-14-10001.

Conflicts of Interest: The authors declare no conflict of interest.

\section{References}

1. Carroll, S.B. Evo-Devo and an Expanding Evolutionary Synthesis: A Genetic Theory of Morphological Evolution. Cell 2008, 134, 25-36. [CrossRef] [PubMed]

2. Irschick, D.J.; Albertson, R.C.; Brennan, P.; Podos, J.; Johnson, N.A.; Patek, S.; Dumont, E. Evo-devo beyond morphology: From genes to resource use. Trends Ecol. Evolut. 2013, 28, 267-273. [CrossRef] [PubMed]

3. Wanninger, A. Morphology is dead-long live morphology! Integrating MorphoEvoDevo into molecular EvoDevo and phylogenomics. Front. Ecol. Evolut. 2015, 3, 54. [CrossRef]

4. Soltis, D.E.; Visger, C.J.; Soltis, P.S. The polyploidy revolution then ... and now: Stebbins revisited. Am. J. Bot. 2014, 101, 1057-1078. [CrossRef] [PubMed]

5. King, M. Species Evolution: The Role of Chromosome Change; Cambridge University Press: Cambridge, UK, 1993.

6. Kramerov, D.A.; Vassetzky, N.S. Origin and evolution of SINEs in eukaryotic genomes. Heredity 2011, 107, 487-495. [CrossRef] [PubMed]

7. Altukhov, Y.P. Intraspecific Genetic Diversity. Monitoring, Conservation and Management; Springer: Berlin/Heidelberg, Germany, 2006.

8. Lynch, M. The Origins of Genome Architecture; Sinauer Associates Inc.: Sunderland, MA, USA, 2007.

9. Shapiro, J.A. Evolution: A View from the 21st Century; FT Press Science: Upper Saddle River, NJ, USA, 2011.

10. Koonin, E.V. The Logic of Chance. The Nature and Origin of Biological Evolution; FT Press Science: Upper Saddle River, NJ, USA, 2012.

11. Parisod, C.; Holderegger, R.; Brochmann, C. Evolutionary consequences of autopolyploidy. New Phytol. 2010, 186, 5-17. [CrossRef] [PubMed]

12. Clarke, J.T.; Lloyd, G.T.; Friedman, M. Little evidence for enhanced phenotypic evolution in early teleosts relative to their living fossil sister group. Proc. Natl. Acad. Sci. USA 2016, 113, 11531-11536. [CrossRef] [PubMed]

13. Benfey, T.L. Effectiveness of triploidy as a management tool for reproductive containment of farmed fish: Atlantic salmon (Salmo salar) as a case study. Rev. Aquac. 2016, 8, 264-282. [CrossRef]

14. Gladyshev, M.I.; Artamonova, V.S.; Makhrov, A.A.; Sushchik, N.N.; Kalachova, G.S.; Dgebuadze, Y.Y. Triploidy does not decrease contents of eicosapentaenoic and docosahexaenoic acids in filets of pink salmon Oncorhynchus gorbuscha. Food Chem. 2017, 216, 66-69. [CrossRef] [PubMed] 
15. Lien, S.; Koop, B.F.; Sandve, S.R. The Atlantic salmon genome provides insights into rediploidization. Nature 2016, 533, 200-207. [CrossRef] [PubMed]

16. Pasquier, J.; Braasch, I.; Batzel, P. Evolution of gene expression after whole genome duplication: New insights from the spotted gar genome. BioRxiv 2017, 151944. [CrossRef] [PubMed]

17. Robertson, F.M.; Gundappa, M.K.; Grammes, F.; Hvidsten, T.R.; Redmond, A.K.; Lien, S.; Martin, S.A.M.; Holland, P.W.H.; Sandve, S.R.; Macqueen, D.J. Lineage-specific rediploidization is a mechanism to explain time-lags between genome duplication and evolutionary diversification. Genome Biol. 2017, $18,111$. [CrossRef] [PubMed]

18. Weise, A.; Mrasek, K.; Klein, E.; Mulatinho, M.; Llerena, J.C., Jr.; Hardekopf, D.; Pekova, S.; Bhatt, S.; Kosyakova, N.; Liehr, T. Microdeletion and Microduplication Syndromes. J. Histochem. Cytochem. 2012, 60, 346-358. [CrossRef] [PubMed]

19. Phillips, R.; Rab, P. Chromosome evolution in Salmonidae (Pisces): An update. Biol. Rev. 2001, 76, 1-25. [CrossRef] [PubMed]

20. Zelinsky, Y.P.; Makhrov, A.A. Homological series by chromosome number and the genome rearrangements in the phylogeny of Salmonoidei. Russian J. Genet. 2002, 38, 1115-1120. [CrossRef]

21. Sutherland, B.J.G.; Gosselin, T.; Normandeau, E.; Lamothe, M.; Isabel, N.; Audet, C.; Bernatchez, L. Salmonid chromosome evolution as revealed by a novel method for comparing RADseq linkage maps. Genome Biol. Evolut. 2016, 8, 3600-3617. [CrossRef] [PubMed]

22. Balon, E.K. The Tao of life: From the dynamic unity of polar opposites to self-organization. In Alternative Life-History Styles of Animals; Bruton, M.N., Ed.; Kluwer Academic Publishers: Dordrecht, The Netherlands, 1989; pp. 7-40.

23. Qumsiyeh, M.B. Evolution of number and morphology of mammalian chromosomes. J. Heredity 1994, 85, 455-465. [CrossRef]

24. Pavlov, D.A.; Osinov, A.G. Main features of early ontogeny in Salmonids (Salmoniformes) and other representatives of the protacanthopterygii in relation to the phylogeny. J. Ichthyol. 2004, 44, 267-283.

25. Pavlov, D.S.; Savvaitova, K.A. On the Problem of Ratio of Anadromy and Residence in Salmonids (Salmonidae). J. Ichthyol. 2008, 48, 778-791. [CrossRef]

26. Artamonova, V.S. Genetic markers in population studies of Atlantic salmon Salmo salar L.: Karyotype characters and allozymes. Russian J. Genet. 2007, 43, 221-233. [CrossRef]

27. Zelinsky, Y.P.; Makhrov, A.A. Chromosomal variability, genome reorganization in phylogeny, and the systematics of Salmo and Parasalmo species (Salmonidae). J. Ichthyol. 2001, 41, 209-216.

28. Frolov, S.V. Karyotype Variability and Evolution in Salmonidae; Dalnauka: Vladivostok, Russia, 2000; (In Russian, Summary in English).

29. Verspoor, E.; Stradmeyer, L.; Nielsen, J. (Eds.) The Atlantic Salmon. Genetics, Conservation and Management; Blackwell Publishing Ltd.: Oxford, UK, 2007.

30. Dorofeeva, E.A. Systematics and distribution history of European salmonid fishes of the genus Salmo. J. Ichthyol. 1998, 38, 419-429.

31. Leitwein, M.; Gunand, B.; Pouzadoux, J.; Desmarais, E.; Berrebi, P.; Gagnaire, P.A. A dense brown trout (Salmo trutta) linkage map reveals recent chromosomal rearrangements in the Salmo genus and the impact of selection on linked neutral diversity. G3 (Bethesda) 2017, 7, 1365-1376. [CrossRef] [PubMed]

32. Jonsson, B.; Jonsson, N. Ecology of Atlantic Salmon and Brown Trout. Habitat as a Template for Life Histories; Springer: Dordrecht, The Netherlands, 2011.

33. Makhrov, A.A.; Ponomareva, M.V.; Khaimina, O.V.; Gilepp, V.E.; Efimova, O.V.; Nechaeva, T.A.; Vasilenkova, T.I. Abnormal development of gonads of dwarf females and low survival of their offspring as the cause of rarity of resident populations of Atlantic salmon (Salmo salar L.). Russian J. Dev. Biol. 2013, 44, 326-335. [CrossRef]

34. Valiente, A.G.; Juanes, F.; Nuñez, P.; Garcia-Vazquez, E. Is genetic variability so important? Non-native salmonids in South America. J. Fish Biol. 2007, 71 (Suppl. D), 136-147. [CrossRef]

35. Yurtseva, A. Intraspecific morphological heterogenicity of Atlantic salmon in the wild and aquaculture. In Salmon. Biology, Ecological Impacts and Economic Importance; Woo, P.T.K., Noakes, D.J., Eds.; Nova Science Publishers, Inc.: New York, NY, USA, 2014; pp. 23-45.

36. Crawford, S.S.; Muir, A.M. Global introductions of salmon and trout in the genus Oncorhynchus: 1870-2007. Rev. Fish Biol. Fish. 2008, 18, 313-344. [CrossRef] 
37. Crespi, B.J.; Teo, R. Comparative phylogenetic analysis of the evolution of semelparity and life history in salmonid fishes. Evolution 2002, 56, 1008-1020. [CrossRef] [PubMed]

38. Markevich, G.N.; Ivashkin, E.G.; Pavlov, E.D. Postspawning survival in lacustrine Sockeye salmon Oncorhynchus nerka Walb. Biol. Bull. 2011, 38, 533-537. [CrossRef]

39. Morita, K.; Nagasawa, T. Latitudinal variation in the growth and maturation of masu salmon (Oncorhynchus masou) parr. Can. J. Fish. Aquat. Sci. 2010, 67, 955-965. [CrossRef]

40. McKay, S.J.; Nakayama, I.; Smith, M.J.; Delvin, R.H. Genetic relationship between Masu and Amago Salmon examined through sequence analysis of nuclear and mitochondrial DNA. Zool. Sci. 1998, 15, 971-979. [CrossRef]

41. Behnke, R.J. Trout and Salmon of North America; The Free Press: New York, NY, USA, 2002.

42. Chereshnev, I.A.; Skopets, M.B. Salvethymus svetovidovi gen et sp. nova. A new endemic fish of the subfamily Salmoninae from the Lake El'gygytgyn (Central Chukotka). J. Ichthyol. 1990, 30, 87-103.

43. Crête-Lafrenière, A.; Weir, L.K.; Bernatchez, L. Framing the Salmonidae Family Phylogenetic Portrait: A More Complete Picture from Increased Taxon Sampling. PLoS ONE 2012, 7, e46662. [CrossRef] [PubMed]

44. Osinov, A.G.; Senchukova, A.L.; Mugue, N.S.; Pavlov, S.D.; Chereshnev, I.A. Speciation and genetic divergence of three species of charr from ancient Lake El'gygytgyn (Chukotka) and their phylogenetic relationships with other representatives of the genus Salvelinus. Biol. J. Linn. Soc. 2015, 116, 63-85. [CrossRef]

45. Alekseyev, S.S. Reduction of skeletal elements in Arctic charr Salvelinus alpinus from Transbaikalia and the origin of some morphological features of Svetovidov's long-finned charr Salvethymus svetovidovi. J. Ichthyol. 2000, 40, 495-504.

46. Adams, C.E.; Maitland, P.S. Arctic charr in Britain and Ireland-15 species or one? Ecol. Freshw. Fish 2007, 16, 20-28. [CrossRef]

47. Noakes, D.L.G. Charr truth: Sympatric differentiation in Salvelinus species. Environ. Biol. Fishes 2008, 83, 7-15. [CrossRef]

48. Klementsen, A. The charr problems revisited: Exceptional phetotypic plasticity promotes ecological speciation in postglacial lakes. Freshw. Rev. 2010, 3, 49-74. [CrossRef]

49. Knudsen, R.; Klemetsen, A.; Alekseyev, S.; Adams, C.E.; Power, M. The role of Salvelinus in contemporary studies of evolution, trophic ecology and anthropogenic change. Hydrobiologia 2016, 783, 1-9. [CrossRef]

50. Chereshnev, I.A. Threatened fishes of the world: Salvethymus svetovidovi Chereshnev \& Scopets, 1990 (Salmonidae). Environ. Biol. Fishes 1996, 46, 166.

51. Phillips, R.B.; Marsuoka, M.P.; Konkol, N.R.; McKay, S. Molecular systematics and evolution of the growth hormone introns in the Salmonidae. Environ. Biol. Fishes 2004, 69, 433-440. [CrossRef]

52. Pankova, M.V.; Brykov, V.A. Divergence of introns in the paralogous growth hormone genes of salmonid fish indicates the effect of selection. Dokl. Biol. Sci. 2013, 451, 231-234. [CrossRef] [PubMed]

53. Crespi, B.J.; Fulton, M.J. Molecular systematics of Salmonidae: Combined nuclear data yields a robust phylogeny. Mol. Phylogenet. Evolut. 2004, 31, 658-679. [CrossRef] [PubMed]

54. Shedko, S.V.; Miroshnichenko, I.L.; Nemkova, G.A. Phylogeny of salmonids (Salmoniformes: Salmonidae) and its molecular dating: Analysis of nuclear RAG1 gene. Russian J. Genet. 2012, 48, 575-579. [CrossRef]

55. Phillips, R.B.; Oakley, T.H. Phylogenetic relationships among the Salmoninae based on nuclear and mitochondrial DNA sequences. In Molecular Systematics of Fishes; Kocher, T.D., Stepien, C.A., Eds.; Academic Press: San Diego, CA, USA; London, UK, 1997; pp. 145-162.

56. Gritsenko, O.F. Diadromous Fishes of Sakhalin (Systematics, Ecology, Fisheries); VNIRO Publishing: Moscow, Russia, 2002. (In Russian)

57. Honda, K.; Arai, T.; Takahashi, N.; Miyashita, K. Life history and migration of Sakhalin taimen, Hucho perryi, caught from Lake Akkeshi in eastern Hokkaido, Japan, as revealed by Sr:Ca ratios of otoliths. Ichthyol. Res. 2010, 57, 416-421. [CrossRef]

58. Honda, K.; Kagiwada, H.; Takahashi, N.; Miyashita, K. Movement patterns of adult Sakhalin taimen, Parahucho perryi, between stream habitats of the Bekanbeushi River system, eastern Hokkaido, Japan. Ichthyol. Res. 2014, 61, 142-151. [CrossRef]

59. Zimmerman, C.E.; Rand, P.S.; Fukushima, M.; Zolotukhin, S.F. Migration of Sakhalin taimen (Parahucho perryi): Evidence of freshwater resident life history types. Environ. Biol. Fishes 2012, 93, 223-232. [CrossRef] 
60. Edo, K.; Kawaguchi, Y.; Nunokawa, M.; Kawamula, H.; Higashi, S. Morphology, stomach contents and growth of the endangered salmonid, Sakhalin taimen Hucho perryi, captured in the Sea of Okhotsk, northern Japan: Evidence of an anadromous form. Environ. Biol. Fishes 2005, 74, 1-7. [CrossRef]

61. Reshetnikov, Y.S. Ecology and Systematics of Coregonids; Nauka: Moscow, Russia, 1980. (In Russian)

62. Ritchie, A.M.; Lo, N.; Ho, S.Y.W. Examining the sensitivity of molecular species delimitations to the choice of mitochondrial marker. Org. Divers. Evolut. 2016, 16, 467-480. [CrossRef]

63. Østbye, K.; Amundsen, P.-A.; Bernatchez, L.; Klementsen, A.; Knudsen, R.; Kristoffersen, R.; Næsje, T.F.; Hindar, K. Parallel evolution of ecomorphological traits in the European whitefish Coregonus lavaretus (L.) species complex during postglacial times. Mol. Ecol. 2006, 15, 3983-4001.

64. Etheridge, E.C.; Adams, C.E.; Bean, C.W.; Durie, N.C.; Gowans, A.R.D.; Harrod, C.; Lyle, A.A.; Maitland, P.S.; Winfield, I.J. Are phenotypic traits useful for differentiating among a priori Coregonus taxa? J. Fish Biol. 2012, 80, 387-407. [CrossRef] [PubMed]

65. Jacobsen, M.W.; Hansen, M.M.; Orlando, L.; Bekkevold, D.; Bernatchez, L.; Willerslev, E.; Gilbert, M.T.P. Mitogenome sequencing reveals shallow evolutionary histories and recent divergent time between morphologically and ecologically distinct European whitefish (Coregonus spp.). Mol. Ecol. 2012, 21, 2727-2742. [CrossRef] [PubMed]

66. Schmalhausen, I. The problem of stability of organic forms (ontogeneses) in the process of evolution. J. Gen. Biol. 1945, 6, 3-15, (In Russian, summary in English).

67. Makhrov, A.A.; Lajus, D.L. Post-glacial colonization of the North European Seas by Pacific fishes and lamprey. Contemp. Probl. Ecol. 2017, in press.

68. Khlebovich, V.V.; Abramova, E.N. Some problems of crustacean taxonomy related to the phenomenon of Horohalinicum. Hydrobiologia 2000, 417, 109-113. [CrossRef]

69. Hugnes, A.L. Evolution of adaptive phenotypic traits without positive Darwinian selection. Heredity 2012, 108, 347-353.

70. Davidson, E.H. The Regulatory Genome. Gene Regulatory Networks in Development and Evolution; Academic Press: Amsterdam, The Netherlands, 2006.

71. Wagner, A. The role of robustness in phenotypic adaptation and innovation. Proc. R. Soc. B 2012, 279, 1249-1258. [CrossRef] [PubMed] 